\title{
Having faith in the online voice \\ Exploring contemporary issues of trust, language and advice in the context of e-health
}

Elizabeth Sillence (Newcastle upon Tyne)

\begin{abstract}
The increasing volume of Internet based health resources means that decisions about how to trust information and advice encountered online become ever more complicated. As peer-topeer experiences become a source of health information, lay people are required to evaluate the trustworthiness of such online personal accounts. In this paper, we present two contemporary studies of the negotiation of trust in e-health. The first study explores how people come to select a trustworthy voice from a community of online peers whilst the second explores how video bloggers use the medium to present a credible account of their health experiences. Drawing on data from interviews with community members, video transcripts and viewers' comments, we examine issues of trust, language and advice from the perspective of those presenting the authentic voice as well as those seeking to evaluate the voice. The paper highlights the importance of similarity matching, motivation and interactivity to the portrayal and recognition of trustworthy accounts online.
\end{abstract}

\section{$1 \quad$ Introduction}

Across a range of conditions, the Internet now acts as a major resource for health (cf. Fox/Duggan/Purcell 2013). Over the last decade, the number of people using the internet for health information and advice has increased. $72 \%$ of adult internet users and $84 \%$ of teens say they have looked online for health information (cf. Fox/Duggan/Purcell 2013; Wartella et al. 2016). There are also more surrogate seekers, those people looking online for information on behalf of someone else (cf. Cutrona et al. 2015). Informal caregivers have also been shown to use the internet for information, support and communication (cf. James et al. 2007).

Seeking information and particularly advice online, however, can be a risky business for health consumers. Faced with an increasing range of Internet based resources, decisions about how to trust the information and advice encountered becomes ever more complicated. The act of trust itself involves the willingness of one individual to make themselves vulnerable to another individual or agency (cf. Brien 1998). In the healthcare context, trust is usually based upon assumptions about the competence and honesty of the healthcare professional, their respect for confidentiality and their willingness to act in the best interests of their patient 
(cf. Hall et al. 2002). The literature around trust in an e-health context also emphasises the importance of a knowledgeable and impartial source of information but in recent years, this source has evolved beyond the domain of healthcare organisations, governmental bodies and charities to include user-generated content. In consequence, individuals as well as professionals and organisations are now engaged in the "art of crafting" trustworthy accounts of healthrelated information and advice.

The trust issues arising from this shift are worth exploring, and central among them is the notion of 'expertise'. Portraying oneself as a credible expert on a particular health topic or role has advanced from being the sole domain of the professional to incorporate that of the lay-person as well. In online spaces, ordinary people are providing accounts of their experiences and offering information and advice to those who find themselves in similar positions, but these roles of advice seeker and giver have to be carefully negotiated. How do those providing the accounts seek to convey their experience and their expertise? Likewise, how do those reading the online accounts respond to and engage with different people, searching, sifting and selecting some personal experiences whilst rejecting others? In this paper, we draw on two contemporary issues in e-health to illustrate these processes. The two studies allow the issues of trust and expertise to be examined from two different perspectives - that of those offering information and advice and those looking for or asking for information and advice.

In the first study, we consider how people making a health decision engage with and select trustworthy accounts from a community of online peers. We consider the strategies people use to sift through online accounts and how they assess trustworthiness in those people "similar to themselves". In the second study, we take health vlogs as source material and examine the ways in which users generating health-related videos seek to convey credible accounts of their experiences, to share their so-called "bro-science" (cf. Hall et al. 2016) with others. In this study, we ask how do people portray trustworthy accounts of personal experience rather than convey messages of self-promotion and product endorsement?

Throughout both studies, the paper seeks to illustrate the ways in which language is used to negotiate and establish a sense of trust between peers. In practical terms, understanding the ways in which people engage with personal narratives of health and wellbeing in comparison to other forms of information and advice has important implications for how resources are created as well as for managing expectations between health professionals and individuals.

Methodologically, the data is analysed using thematic analysis (cf. Braun/Clarke 2006) adopting a contextualist middle ground epistemological position (cf. Tebes 2005) where human actions and experiences can be understood in terms of their social context whilst also recognising the value of the reality of the individual experience. This approach allows us to examine both the ways in which credibility is portrayed and assessed by people engaging with online accounts of personal health and wellbeing. Across both studies, we are working with English language data. Whilst study 1 draws predominantly on data collected from a UK sample, the data in study 2 represents a broader cultural base. We will give further pointers on methodological steps and data selection processes in Section 2 and Section 3 below. 


\section{$2 \quad$ Study 1: Finding the trustworthy voice in a community of voices}

There is a growing body of evidence suggesting that online personal experiences or narratives are useful in terms of health decision-making (cf. Entwistle et al. 2011; Ziebland/Wyke 2012). Decision-making activities occur at a number of distributed points in both time and space (cf. Rapley 2008). Distributed decision-making necessarily involves multiple stakeholders as well as access to a range of different information sources. Online support groups (OSGs) are one such important source of health information. These groups are recognised as being key places for information exchange as well as for social and emotional support offering people a sense of empowerment (cf. Mo/Coulson 2012; van Uden-Kraan et al. 2008). These groups are places of interactive information exchange where people can read each other's experiential accounts, post their own experiences and also engage in advice exchange around decisionmaking (cf. Huber et al. 2011; Sillence 2010). The interactivity is important in this respect, and differs from other forms of personal experience online that show curated, but noninteractive accounts of health. The fact that OSGs by their nature provide people with access to a wide range of other people and experiences means that there are decisions to be made about who to trust and which experiences and advice to value above others.

Advice has been defined as "opinions or counsel given by people who perceive themselves as knowledgeable, and/or who the advice seeker may think are credible, trustworthy and reliable" (DeCapua/Dunham 1993: 519). Exchanging advice and information within online health groups therefore raises interesting issues associated with trust and expertise. Asking for advice and information about a decision puts the asker in a potentially vulnerable position, and various linguistic strategies are used to mitigate this process including humour, politeness and positioning statements (cf. Harrison/Barlow 2009; Locher/Hoffman 2006; Sillence 2010). Displaying sufficient expertise to offer advice can also be problematic, and the way in which this process is handled is dependent to some extent on the ethos of the community. The degree of directness of advice may vary, and members may use mechanisms such as personal experiences to establish expertise and negotiate advice exchange (cf. Kouper 2010; Sillence 2013).

In order to understand how people evaluate trust in OSGs, we draw upon a source of data collected to examine the influence of online resources in decision-making more broadly (cf. Sillence/Bussey 2017). The data consisted of interviews with 18 people (males $=7$ ) aged 27-66 (mean $=49.33$ years). All participants were British with the exception of one male participant who was based in the US. The participants took part in individual interviews either face to face or by telephone and interviews lasted between 30 minutes and 1 hour. All interviews were audio recorded and transcribed. The participants had experience of five focal health issues. These issues represent a range of chronic, acute and stage of life health conditions and were considered likely to cover a breadth of decision types from treatment and procedural decisions, through service provision, lifestyle and screening issues. Focusing on multiple conditions allows a more comprehensive overview of the role of OSGs in decisionmaking. The participants were all registered users of OSGs and typically used one main forum in relation to their decision-making around the particular health issue. Participants interacted with the forums reading and posting messages on the site as well as responding to other posters' questions when they felt able to do so. Being familiar with the site, its members and 
its ethos was an important element of continued use for the participants and it was against this context that the interview covered issues of trust and credibility. Participants were asked about their experiences of using the OSGs and we sought to explore how participants navigated through the personal experiences they encountered within the OSGs and to understand how they decided which accounts to engage with further. We also examined whether participants felt that OSGs offered credible, genuine voices that were useful to them.

Data were thematically analysed (cf. Braun/Clarke 2006). We began by reading and extracting all sections of the transcript that made reference in some way to the notion of trust and credibility. We then read these extracts and coded simple descriptive concepts before grouping together similar ideas under higher order themes. We ended up with three key themes that captured the data: (1) experiences are genuine, (2) evaluation through personal relevance and (3) providing challenge to trustworthy accounts. These three themes are described below and are illustrated with relevant quotes from the data.

\subsection{Accepting that these experiences are genuine}

Whilst participants acknowledged there was a risk that posters may not be genuine, the majority of participants considered the experiences and by implication the posters they had encountered on OSGs were authentic. For some people, this reflected their general disposition. They took the posts at face value, and assumed that people were telling the truth. For others, they relied on the longevity of their involvement with the OSG, their ability to judge the style and tone of the messages and cues relating to the health issue itself.

(1) It depends on the forum [as to how credible the posters are] cos some of the forums I went to when I wanted to find out what my symptoms were and there everybody puts their age and where they come from and what drugs they've tried and what drugs they are on so like they almost have like their medication history on it so you can kind of see what stage they're at and their condition and compare it to yours so for example... but in terms of what they actually write it's mostly just you know I was on these meds and... these ones are better but you don't know whether or not that's true or... it's hard because there doesn't seem to be any kind of checks and how can you verify someone's identity on a forum like that (Participant 6 , female)

(2) [A newcomer] will get half a dozen welcome messages as well as people with relevant experience themselves indicating what their course of action or their treatment plan might be and how things might shape up and I think those people that post regularly are very sensible and quite measured in what they say and there's no scare mongering or no ridiculously incorrect recommendations going out typically and if someone does get something wrong and someone else will chip in fairly quickly and point out the possible error of their ways you know (Participant 2, male)

For a few participants, their relationships with posters had extended beyond the forum and they had met offline as well. This information was offered as a way of illustrating the potential value of OSGs but it also says something about the authenticity of posters - the posters are "real" enough to meet face-to-face. Two participants specifically mentioned the difficulty in knowing who was posting on the forums and one explained how she was aware of an imposter incident within the OSG she used. 
(3) You don't know who they are I mean anyone can sign up can't they? I mean we have had a few people who have been somebody different to who they've said they've been... Yeh there have been a few over the years that I've been there yeh... I don't know [how people find out that they aren't who they say they are] I think when things people say don't add up people report them to the moderator and there have been a few that have come have been on other forums as well you know and done the same sort of thing I think there was girl a few years ago that did it and it turned out she was I dunno just a young student or something and she was just making all this stuff up and they found out through liaising with other forums the moderators... but people were really angry about it it's amazing how angry people were, the regulars I suppose they feel a bit you know violated (Participant 10, female)

Despite this, participants overall were comfortable with the authenticity of the experiences and the posters on the OSGs they used.

\subsection{Understanding personal relevance as a way of evaluating trustworthy accounts}

In the following three examples we see how people describe the process they go through when assessing the credibility of the poster and their message. It is interesting to note that the three examples move from a rational well-argued account of 'relevance' - couched in terms of physiological matching -, through to the less well defined or specified concept of 'resonance' and finally to an explicit admission of the way in which personal narratives are rejected if they offer an alternative perspective from that of the participant.

(4) I give more weight to advice that's from people I know are similar to me i. e. younger, males first, especially ones my own age. You know I'm 44, I was 41 [when making the decision] and there's a place where you can put your age, sex and what not on the website and people can read it. Some people post that information on other places on the website but a lot of people don't - probably the majority of people don't - but it seems a lot of guys, like me, just post it out there cos I don't care, like yeh I'm a dude, I'm 44 but when it comes to this surgery it is relevant whether you're a 40 year male or a 65 year old female. It's just a completely different fact set so I would give more weight to information that had been posted by males particularly ones my own age. (Participant 9, male)

(5) Well I think that comes down to your own personal... what resonates with you, what feels right for you as to whether you take something like that on board but it depends what the advice is. (Participant 13, female)

(6) Yeh I think I just take from it... well stuff that I agree with I take on board and stuff that I don't agree with I just push aside. I think at that point, when I first posted, I think my first question was probably something like have you ever had to make a decision about whether or not to terminate a pregnancy? Cos obviously I was told that there was absolutely no chance of the baby surviving, best case scenario he would survive for a couple of hours after he'd been born I think there's like one baby that lived for 5 years or something but it was completely nothing I mean total life support really, really not great but once you start googling it you do come up with a lot of American sites basically that are 'my baby lived for two months' and things like that so I am a bit like should I be making the decision even though I know he's not going to survive should I even if it's for a day a 
week or something so I think that was it but to be honest I'd kind of made my mind up and there was absolutely no way I was going to put myself and my family through that or a baby to be fair but I dunno I think I was just looking for affirmation that my decision was the right decision but of course it was the right decision for me I realise now there was never any doubt about it but I was just looking for some sort of backup. (Participant 12 , female)

In example (4), the participant describes the importance of sifting through personal experiences on the basis of age and gender. These characteristics offer a certain physiological credibility to the advice of the posters. The participant explains why experiences or advice written by women does not warrant as much "weight" as information and advice from men of the same age. Their expertise is less relevant. The sense of finding someone similar is also expressed in example (5). In this instance, the specific nature of the similar characteristics is not expressed explicitly rather the importance of reading a message or piece of advice that in some way resonates with the reader is described. Here the focus is on the message itself rather than on the poster directly. In example (6) there is a clear admission that experiences are rejected if they are not in line with the participant's own perspective, and the participant screening experiences for those that affirm her worldview.

These examples highlight the important processes involved in meaningful engagement with patient experiences. For participants, it was important to know who was making the contribution. Personal narratives reveal something about the person posting the experience. When engaging with online narratives, participants were quick to reject experiences that come from an individual who seemed different from themselves either because that person came from a different gender, age group, region, or demographic, or because their health profile or severity of condition was a poor match. Example (5) illustrates the importance of assessing "what is being said". Similar findings have indicated that people reject information offered by their peers if it does not resonate with their own understanding of a condition, or if the mixture of information, advice and support on offer was unbalanced or a poor match to their needs (cf. Sillence et al. 2014). Comparing the online narratives with their own experiences, participants sought to assess how credible the material was. Whilst some people saw clear and direct comparisons between their situation and the online experiences, information offered by peers was pushed back if it did not resonate with their own concept of the health condition or challenged their worldview (cf. Sillence et al. 2015). In previous work, we have observed the way that posters within online health groups appear to seek out very like-minded people and employ a variety of discursive techniques to deal with uncongenial advice (cf. Sillence 2010). Like-minded people provide support and reinforcement for pre-existing views allowing only the advice that the poster wants to hear to be prominent in the discussion. In example (6) above we have explicit acknowledgement of that activity occurring with the participant describing how she rejects unpalatable opinions or information stuff that I don't agree with I just push aside. The sense that this participant sought affirmation of her own position is consistent with self-verification theory (cf. Swann/Read 1981), which explicitly predicts that people will seek the advice of others whose experiences and beliefs overlap with their own. 


\subsection{Challenging "trustworthy" accounts}

In examples (7)-(8), we see a more active challenge to the online advice with participants checking and testing both the information contained in the message as well as the poster themselves. In example (7) the participant emphasises the lengths she goes to when checking the information from online peers. Here we can see that the participant notes the value in providing references and other forms of corroboration but that those trust cues in and of themselves are not sufficient. Participants report that they have actively followed up references and made checks on other websites. So in example (7), the participant uses many action verbs demonstrating the scope and the effort of the corroboration process that takes place before any information is deemed trustworthy and accepted (check, click, pick and choose, clarify, double check).

(7) Yes I check and I mean obviously there's a lot on the web and some people link it to sites you know so you can then read that some people you know say link a study or something so you can just click on the link which is very helpful... I think the important bit is to pick and choose and also to clarify I often double check things so I go on different things to see if that's what other people are saying I don't think sometimes you can accept just one thing I think you need to see that its consistent against different things you know against different groups I think that's important. (Participant 11, female)

In example (8), the participant describes a time when she was concerned about the results of a pregnancy test and turned to her online support group for advice. Rather than providing her own personal experience as a way of asking for advice (cf. Sillence 2013), she deliberately chooses to keep the discussion hypothetical to remove the possibility of receiving biased responses rather than telling me what they think I want to hear.

(8) I remember when I found out I was pregnant with XXXX that I had a really faint line and I was just convinced that it wasn't going to work out again and I went in the miscarriage section and I posted does anybody know... does anybody have experience of having faint lines you know faint positive tests in the early days does that make you any more likely to have a miscarriage? I just wanted to see what people came back with and I didn't actually say I'd had it... I just wanted to see what people said... I was just like I just thought I'd post it without actually saying my situation like a theoretical thing and see what people actually said so I'd maybe get the real... rather than telling me what they think I want to hear. (Participant 10, female)

The participant goes onto describe how she received a reply from a poster suggesting that faint lines on a pregnancy test were likely to lead to miscarriage. However, later on the participant provided an update in her diary (this is an online diary embedded within the website - it runs alongside the forum facility and operates as an modifiable profile) and the same poster got back in touch to express her shock and regret. She described the fact that she had not known that the participant was detailing her own situation - she had not realised the significance of your post indicating that she would have given a more mitigated answer if she had known that the participant was not talking hypothetically. The participant then says she wrote back to the poster explaining why she hadn't been open about her own situation and observes that sometimes in this group, if you let them, people tell you what they think you want to hear that just showed me you know really that people are biased even the ones that you probably 
trust you know. Again it may be that despite talk of unbiased information and real information that in this case the participant was ultimately still concerned with a need to seek out likeminded people for support and reinforcement.

When faced with information from an organisation, a key user requirement in terms of trust is that the site is unbiased. The very nature of peer-to-peer resources, however, means that advice and experiences will necessarily show a particular viewpoint, which will not be neutral or impartial in the same way that organisations attempt to be. Whilst the offering of advice couched in personal experiences often makes explicit that such advice is "just what happened to me", or "is only my experience" (cf. Sillence 2013; emphasis added), it is clear that bias and impartiality are less clearly defined and identifiable terms when used in relation to personal experiences. The ethos of the community and the linguistic devices employed by members will dictate the extent to which bias is accepted as an inherent feature of individual accounts or is seen as a well-intentioned consequence of the prevailing social norms.

The examples presented here demonstrate the way in which participants take a critical and often active approach to the assessment of the information and advice contained in the forums. The majority of participants in this study had considerable knowledge about both their health condition and the practices of the online groups they used. It may be that other people using OSGs, in particular those who are less knowledgeable about the health topic or who are less actively engaged with the forum, may not be so well equipped to assess the credibility of the experiences and the posters they encounter.

\section{Study 2: Presenting a trustworthy account of experience and motivation in online videos}

In study 1 , we established that personal narratives are welcomed by many as providing a genuine, authentic voice online. In this second study, we focus specifically on the personal narratives themselves and examine the kinds of cues posters use to create a sense of credibility around their accounts online. Recognising the contemporary use of video based personal narratives, the second study takes YouTube videos as is source material.

YouTube, founded in 2005, allows users to post, view, comment on and link to videos on the site. Video is an increasingly common medium for users wishing to capture and share their experiences in a health context. These videos can exist as standalone, single narratives focussing on a particular health topic, product or service or as a form of journaling video blog commonly referred to as vlogs (cf. Burgess/Green 2009). Health vlogs, for example, typically focus on chronic or longer term health conditions and document the ongoing lives of their creators (cf. Godwin-Jones 2007). Vlogs may fall under a number of different genres including teaching, personal journal and self-documentary (cf. Liu et al. 2013) and self-reflection and altruism may be key drivers for their creation (cf. Huh et al. 2014; Wotanis/McMillan 2014). Whilst many health videos document the day to day living of someone with a certain health condition, videos that refer to particular treatments, products or services can potentially be seen as testimonials i. e. owned or contrived by a third party with a vested interest (cf. Hohm/Snyder 2015; Vance/Howe/Dellavalle 2009) and user generated videos that contain a strong commercial flavour may be viewed with scepticism (cf. Sillence/Hardy/Briggs 2013). The potential power of these accounts has been recognised by commercial organisations and

ISSN 1615-3014 
personal experiences are now spreading beyond their traditional base of OSGs and are being captured and re-imagined in more professional and corporate settings. This presents a problem for individuals wanting to present trustworthy, credible accounts of their health experiences. They need to distance themselves from potential accusations of self-promotion and product endorsement whilst still portraying a convincing account of their own motivations.

In order to explore what kinds of cues video posters use to create and convey credibility we explore data taken from a larger data set (cf. McNeill/Sillence under review) collected to explore vloggers' motivations for sharing more broadly. The overall corpus consists of 72 videos taken from YouTube. All videos were in English but represented posters from a range of countries including the UK, USA, Australia and India. All videos had been classified by two coders as being examples of product users talking directly to camera in an unscripted fashion. This coding definition was to denote "bro-science videos" (cf. McNeill/Sillence under review). This concept has received little academic attention but is often portrayed in the media in a derogatory manner to refer to the sharing of information and ideas of questionable scientific credibility among lay peers (cf. Hall et al. 2016). Bro-science is often discussed in relation to body-building but here we argue that the notion has relevance in the exchange of experiential information and advice around the topic of male hair loss and the products used in relation to this problem. In labelling these videos as bro-science we seek to identify them as a distinct group, separate from professional product seller videos and as such adopt a neutral stance towards the concept. In addition to the videos we examined the first page of online responses to each of the videos in order to provide information about how the credibility of the video was perceived by others. All videos are publicly available but in keeping with the British Psychological Society's (2017) guidance on mediated research we have not made the video itself identifiable, have refrained from including usernames and have deleted any identifiable information (e. g. location details). To avoid overt product placement all product names have been replaced with Product 1,2 etc.

We selected two videos from the overall corpus one representing each of the two predominant types of video present. The first is an example of a journal or diary style video and the second represents a single, stand-alone video. Both videos displayed patterns of credibility cues we saw present in the larger corpus, including reference to the body as evidence, the documenting of first-hand experience, the characterisation of "like-minded" and the implicit or explicit rejection of financial motivations. Patterns in the data were identified by viewing each video and asking "In what ways is the poster conveying credibility?" Relevant sections of the video were then transcribed and labelled with a code (credibility cue). These codes were then grouped into themes. In addition, the online responses to the videos were also considered. The first page of responses was examined and comments that related to the credibility themes identified were transcribed. Their function in relation to accepting or refuting the credibility claims was then described.

\subsection{Video 1}

This is the third in a series of videos made by the poster to document his experiences with Product 1 a topical treatment for hair loss. 


\section{Establishing a sense of the like-minded}

In the video, the poster is sitting in his bedroom at a computer desk talking directly to the camera. The homely setting provides a backdrop for what is intended to be an authentic account of his experiences over the last few months. Rather than striving for a highly polished professional video, the poster is content to talk off-topic about, for example, his wife and his weight loss. This authenticity is aided by the poster's technical problems and his difficulty in remembering the point he was hoping to make (examples (9)-(10)).

(9) I'm going to stop this video before this camera goes haywire I'm starting to see flashes all over the place on the computer you know warning me of the temperature of the camera.

(10) What else? [long pause] oh crap man I totally forgot what I was going to say

There is no sense that this is a scripted or polished production, edited or re-shot or completed to a specific timeframe. Despite indicating that he wants to keep the video short to please the viewers (Let's skip quickly through this video cos I know you guys don't like to see 10, 20 minute videos cos those suck), the video runs to over 9 minutes long. This amateur style of delivery, we argue, conveys a sense of credibility through authenticity (cf. Metzger/Flanagin 2015). The poster is not a professional in any sense but a regular guy, simply reporting on his experiences. The technical difficulties he encounters reinforce the idea that he is a "real" person and again this adds credibility (cf. Fogg/Tseng 1999). Here the amateur rather than the more professional presentation acts as trust cue in terms of developing the authentic voice. The poster is conveying his lay experience to viewers and is able to present himself as someone who thinks and behaves in a like-minded manner. As with study 1, we see the importance of conveying a sense of similarity between those who are providing the information with those who are seeking or viewing the information. This contrasts with professional product sellers who maintain distance and "otherness" between themselves and those seeking information in order to re-inforce the information differential.

\section{Documenting first-hand experience}

In example (11) below, the poster uses the video to communicate the product benefits. Here the important thing about the review is that the user has tested the product on himself, which gives him the authority to talk about the product. He discusses the efficacy of the product and his surprise that the product started working so quickly. He also draws the distinction between the product claims and his own experience of using the product. The fact that he has been surprised and has been lucky suggests that this was not expected or planned in anyway again this helps to portray an experience that has exceeded expectations rather than one that has simply conformed to some preconceived "plan". This information might resonate with advice-seekers who do not expect the product to work either.

(11) I have been on Product 1 for 4 and half 5ish months and the response has been good it looks like this thing is working - you can't expect too much in 4 and half months it says it takes 6 months to start kicking in but with me it started right away so I hope anyone on the site starts to use it has the same luck that I do 
Later the poster discusses the downsides of using the product and raises the issue of side effects (example (12)). Rather than play down the negatives, he presents them in graphic detail (shedding, I lost a lot of hair, peeling from the skin). Detailing the experienced side effects associated with the product help distinguish it from being a straightforward marketing video, and this further establishes the credibility of the poster.

(12) By far the most asked question is side effects what kind of side effects do you have well like I said before, the first two, three weeks I had a lot of shedding, a lot of shedding I lost a lot of hair. Aside from that with it came a little bit of peeling from the skin. It dried the whole area off and peeled and that happened twice within that period of two to three weeks. So aside from being the bald guy the super bald guy in the company I was also the guy that shed flakes that didn't look good that was for a whole frigging three weeks of not wearing anything black...

\section{The body as evidence}

In example (13) we see a commentary around a key feature of the bro-science genre (as identified by McNeill/Sillence under review) - that is the way in which it presents visual evidence for claims. Here the poster invites the viewers to compare his current hair growth with images taken some time before. He is asking them to judge for themselves the evidence of his experience and his claims about the product.

(13) At the end of this video I am going to have a picture - a kind of before and after deal....the after is going to be right here sitting at this desk I'm just going to tilt my head a little over here and you can see I'm almost done and the before is going to be from any of the days that I took pictures from wherever I was I will try and find a picture that shows my head and how deeply I was getting into baldness.

\section{Rejection of ulterior motives}

The poster ends his YouTube video by encouraging people to also view his open profile Facebook page for further photos documenting his hair loss and regrowth. He thanks those who have already viewed his Facebook page. By referring to his Facebook page he is also able to explicitly reject the notion that he is gaining financially from the product and he disavows any financial stake in making these videos.

(14) Please tell me what you think about the photos and thanks for looking at Facebook. You guys have seen it's not a Facebook page to sell stuff it's just me having a good time.

The fact that this poster has a presence on multiple platforms is important in terms of the way he portrays his credibility. Facebook profiles are usually assumed to be authentic and as such the poster is pointing viewers to another credibility cue. One that will both enhance his credibility as a like-minded person, provide further "evidence" of his hair growth and provide support for his rejection of financial motivations. 


\subsection{Viewers' responses to the video}

In the comments beneath the video, viewers recognise and in general accept two key trust cues. The first is the "visual evidence" of the product's efficacy and the second relates to the authenticity of the poster as a genuine, everyday guy.

In examples (15)-(16) the viewers recognise the use of the "before and after" premise as "proof" of the product's efficacy. Both comments indicate that the viewers now believe that the product works.

(15) great results proof that product 1 works!

(16) Hey, thank you man! I came across your earlier video and actually waited for this time when you'd (hopefully) show results. And you did! Thanks a lot! Your vid is very helpful, quite reassuring. Hopefully, this thing will work on me! Once again, I appreciate your posts. Have a great one!

The poster's video works hard to portray a normal everyday guy, who has managed to make time to produce this video despite the obligations of daily life. This effort is recognised and welcomed by some but not by others as the three examples (17)-(19) below illustrate.

(17) Thanks for the effort. This has been very informative and helpful. You seem like a cool guy.

(18) Thanks for your positive video(s)-( both my friends and I appreciate your efforts in making your informative videos re: Product 1 products.

(19) the worst review ever dude get to the point and state the facts we don't need to know your life story

In example (19) there is no sense that the poster is viewed as anything other than trustworthy but that the very cues that add to the credibility of the personal experience can be in tension with the genre of product review. Here this viewer dislikes having to watch a 9-minute video to find out whether or not the product works.

\subsection{Video 2}

This is a single video in which the poster is sitting at an office desk talking directly to a webcam about his experiences using Product 2 (this is a brand name of the generic Product 1).

\section{A sharing motivation}

Once again the main tone of this bro-science video is the ordinary and unremarkable nature of this poster describing his everyday experiences with the product. He suggests that he simply wants to share his experiences with the viewers, and he demonstrates this through the casual way in which he introduces the video and talks about his motivations for sharing (examples (20)-(22)). In this case, the use of the word just presents sharing experiences as normal and mundane (emphasis added):

(20) I just thought I'd do a video on my experience with Product 2

(21) Anyway I just thought I'd give my review of the stuff 
(22) Anyway I thought I'd just let you know

By conveying his motivations for sharing, the poster is attempting to convey the credibility of his experiences. People often rely upon heuristics, or simple rules of thumb, to help them assess the credibility of online information. The persuasive intent heuristic (cf. Metzger/Flanagin 2013) is one way in which people assess credibility online by checking the source and the message itself for signs of ulterior, possibly nefarious motivations. In a health context, commercially driven motivations are particularly damaging for credibility.

\section{Documenting first-hand experience}

The poster presents his credentials in this area by discussing his previous track record with other hair loss treatment products (examples (23)-(24)). By demonstrating his knowledge of the products, he not only builds up his expertise but can be seen to be helping the viewers by saving them time on products he has already tried and tested on their behalf.

(23) I've recently gone onto this Product 2 extra strength and prior to this I've used Product 1 the standard strength and I've also used Product 3 which is like an anti-cancer medication so it helps with DHT stuff and prevents hairloss but I wouldn't recommend the Product 2 or Product 1 this stuff however has actually worked for me

(24) As I've said I've used other ones before that didn't do anything but this stuff works. It said that it would work in a month or a month and a bit. I actually noticed regrowth in two weeks so that's how happy I am with it

\section{The body as evidence}

In example (25) the poster uses the medium to provide visual evidence. The poster is happy for the viewers to "see" that he knows how to use the product and he demonstrates his expertise in using the product.

(25) So what I do is, I get up in the morning, and I put a little sorta dot there, and a dot there, twice on both sides and then I'll put a line down the top of my head. You just rub it in and leave it in. You use it once morning and night and then go to bed.

\subsection{Viewers' responses to the video}

In the comments, viewers contest two key trust cues. The first relates to the way the poster documents his first hand experience of using other hair loss products, and the second relates to the one-off nature of the video.

Product 2, the product reviewed in this video, is a brand name of the more generic Product 1 . In example (26) the viewer challenges the poster's knowledge and hence his credibility by suggesting in no unclear manner (you fucking retard!) that the poster is confused about the different products he claims to have used. Whilst it is possible that the poster has used both the generic and the branded product in different strengths, the fact that he did not make this explicit undermines the credibility of the poster in this viewer's eyes.

(26) You said in your video that you don't recommend product 1 but you recommend product 2. Product 2 IS Product 1 you fucking retard! (commentator 1)

ISSN 1615-3014 
Unlike the first example, this video is a single video rather than one of a series of updates. This gives viewers the opportunity to question the veracity of the claims made by the poster. In example (27) we see the motivations of the poster being challenged. In fact, the viewer is suggesting that far from providing a bro-science video, this is in fact a marketing video on behalf of the product company.

Interestingly, we see the original poster reply to the comment refuting the accusation (example (28)).

(27) You are just doing advertising for the company, prove me wrong, it's been a year since you show us that video... show us the current results (commentator 2)

(28) no I'm not actually this is a genuine product review. The stuff does work but you have to put it on every day. It doesn't work for everybody, and it did actually say it didnt work for my particular type of hair loss on the box which i found out later, but i still managed to regrow a significant amount of hair. I have actually stopped taking it because of the cost of it, and im not sure its good to take something like this long term. So my hair has actually receeded again somewhat. Takes a long time though for it to fall out again but you will notice thinning when you stop taking it. (original poster)

(29) Its 4 months for $\$ 30$. If it really worked you wouldn't have stopped. Can you show this 'significant' growth you got? no you cant. (commentator 3)

The original poster denies the accusation that this video is anything other than genuine. Once again, he points to the fact that the product worked despite a number of potential barriers (doesn't work for everybody and not my particular type of hair loss) - it exceeded expectations. He provides a justification for stopping using the product in terms of cost and concerns for his health. The notion that using the product over the longer term may not be a good idea, although not followed up again in the discussion, helps to refute the accusation of marketing the product, thereby giving further credibility to his authenticity as a "normal" user of the product, who shares health concerns with his viewers. The poster also plays down the current situation contrasting the original significant amount of regrowth with the somewhat of the now receding hairline.

Although the poster refutes the accusation of a financial motivation and provides a reasoned argument as to why he is no longer using the product, this does not stop other posters from suggesting that he is unable to "prove" this growth. In example (29) commentator 3 rhetorically asks the poster to prove the effectiveness of the product and uses direct quotes from the poster's own rebuttal significant to draw attention to the disparity between what the poster is saying and their lack of evidence.

\section{Discussion}

This paper has shown how those providing information and advice about health online construct a credible voice. It has also illustrated how those searching for information and advice interpret, accept or challenge the cues provided.

The two studies in this paper, the first on online support groups and the second on video blogging, highlight a number of different issues in the search for an authentic voice. Firstly, in 
order to assess credibility, people put importance on similarity matching. Participants examine cues as to the poster's age and gender before then examining the content of their message for resonance in terms of experience, attitude or decision-making. In selecting accounts, participants are looking for accounts that are not just overtly credible in terms of trust cues but personally meaningful for them. It is these accounts that, after verification, they are perhaps prepared to act upon. The concept of homophily is important here in relation to potential behaviour change. Homophily addresses the extent to which two or more individuals who interact are similar in certain attributes, beliefs, education, social status, and preferences (cf. Rogers 2003). Homophily increases the source credibility of information (cf. Phua 2016), which in turn may encourage greater acceptance of messages (cf. Hovland/Weiss 1951).

Secondly, in order to portray credibility, we note the importance of crafting and communicating motivation within eHealth settings. The video posters were keen to stress the genuine nature of their experiences distancing themselves from potential accusations of financial gain in relation to the products they were using. These motivations were challenged by viewers and threatened the credibility of the poster's claims. In this respect, our findings reflect recent observations that commercial overtones act as a barrier to trust in online personal experiences (Sillence et al. 2013).

Although motivations were usually accepted by OSG participants, a tension still existed between the need to read genuine thoughts and experiences and the norms of the community in providing a positive outlook in the messages. This underlying sense of bias or impartiality, although more difficult to define in the context of individual experiences remains an important marker of trust within e-health resources (cf. Blythe/Sillence/Briggs 2017).

Finally, in examining the two examples side by side we see the importance of the medium in dictating the way in which information and advice exchange takes place and consequently the way in which trust is negotiated. Online health communities offer an interactive place for advice exchange in relation to decision making (cf. Sillence/Bussey 2017), allowing members to question and explore the personal experiences and the advice they receive. These sort of negotiations take place over time within the context of the OSGs social norms. The ethos of the community, its moderator(s) and members all contribute to an understanding of how people are able to accept some voices and politely reject or ignore others. The broadcast nature of YouTube, however, creates a different environment and one that places a greater onus on the poster to create an authentic voice from the outset. Negotiating trust is something that is more difficult to achieve in this medium with fewer communication norms and a heavier commercial presence. On YouTube the information and advice is, at least initially, offered to all rather than requested by individual members. The broadcast poster therefore has to present a more comprehensive, generic trustworthy message up front and in fact may only have a single opportunity to craft their account. It is important then to note that although the video provides visual evidence, the embodiment of proof (cf. Mazanderani/O'Neill/Powell 2013), the accompanying language and commentary must be supportive, working hard to convey motivations, genuine experience and anticipate queries and challenges. 


\section{Conclusion}

The changing nature of online health resources presents increasing opportunities for people to share their own experiences and offer others information and advice. The range of peer narratives available also presents a challenge in terms of portraying and recognising a trustworthy account. Traditional trust cues such as markers of medical expertise appear to be less relevant than they used to be in this domain. Lay experts must carefully construct a convincing account of their credentialed expertise through detailed profiles, knowledgeable accounts and impartial advice. For advice seekers there is no single set of reliable cues, they are subjective and as such open to challenge and verification and this takes place in different ways depending on the nature of the medium and the ethos of the community. These studies have added to our understanding of the way in which credibility is both portrayed and perceived by those engaged in the exchange of online health information. Both data sets provide interesting perspectives, and whilst a combination of the two i. e. naturalistic, uncensored narratives alongside in depth interviews would have merit, it may prove logistically and ethically difficult to achieve.

Going forward, we are increasingly interested in the use of video-based narratives as a way of combining both visual and textual information and suggest that these data sources, in particular those which record ongoing health conditions over a longer time period, warrant further investigation across a number of health domains.

\section{References}

Blythe, John M./Sillence, Elizabeth/Briggs, Pam (2017): "The role of trust in eHealth websites: The enduring power of impartiality". Frontiers in Public Health. Conference Abstract: 3rd UCL Centre for Behaviour Change Digital Health Conference 2017: Harnessing digital technology for behaviour change. doi: 10.3389/conf.FPUBH.2017.03.00056.

Braun, Virginia/Clarke, Victoria (2006): "Using thematic analysis in psychology". Qualitative Research in Psychology 3/2: 77-101. doi: 10.1191/1478088706qp063oa.

Brien, Andrew (1998): "Professional ethics and the culture of trust". Journal of Business Ethics 17/4: 391-409. doi: 10.1023/A:1005766631092.

British Psychological Society (2017): "Ethics guidelines for internet-mediated research". INF206/04.2017. Leicester. https://beta.bps.org.uk/news-and-policy/ethics-guidelinesinternet-mediated-research-2017 [21.06.2017].

Burgess, Jean/Green, Joshua (2009): YouTube. Online Video and Participatory Culture. Cambridge/Malden: Polity. (= Digital media and society).

Cutrona, Sarah L./Mazor, Kathleen M./Vieux, Sana N./Luger, Tana M./Volkman, Julie E./Rutten, Lila. J. F. (2015): "Health information-seeking on behalf of others: Characteristics of 'surrogate seekers"'. Journal of Cancer Education 30/1: 12-19. doi: 10.1007/ s13187-014-0701-3.

DeCapua, Andrea/Dunham, Joan F. (1993): "Strategies in the discourse of advice". Journal of Pragmatics 20/6: 519-531. doi: 10.1016/0378-2166(93)90014-G.

Entwistle, Vikki A./France, Emma F./Wyke, Sally/Jepson, Ruth/Hunt, Kate/Ziebland, Sue/Thompson, Andrew (2011): "How information about other people's personal experiences can help with healthcare decision-making: A qualitative study". Patient Education and Counseling 85/3: e291-e298. doi: 10.1016/j.pec.2011.05.014. 
Fogg, B. J./Tseng, Shawn (1999): "The elements of computer credibility". In: Proceedings of ACM CHI 99 Conference on Human Factors in Computing Systems. New York, ACM Press: 80-87. doi: 10.1145/302979.303001.

Fox, Susannah/Duggan, Maeve/Purcell, Kristen (2013): "Family caregivers are wired for health". Pew Internet report 2013. www.pewinternet.org/2013/06/20/family-caregiversare-wired-for-health [21.06.2017].

Godwin-Jones, Robert (2007): “Digital video update: YouTube, flash, high-definition”. Language, Learning \& Technology 11/1: 16-21. doi: 10125/44083.

Hall, Mark A./Zheng, Beiyao/Dugan, Elizabeth/Camacho, Fabian/Kidd, Kristin E./Mishra, Aneil/Balkrishnan, Rajesh (2002): "Measuring patients' trust in their primary care providers”. Medical Care Research and Review 59: 293-318. doi: 10.1177/1077558702 059003004.

Hall, Matthew/Grogan, Sarah/Gough, Brendan (2016): "Bodybuilders accounts of synthol use: The construction of lay expertise online". Journal of Health Psychology 21/9: 19391948. doi: 10.1177/1359105314568579.

Harrison, Sandra/Barlow, Julie (2009): "Politeness strategies and advice-giving in an online arthritis workshop". Journal of Politeness Research 5/1: 93-111. doi: 10.1515/ JPLR.2009.006.

Hohm, Carly/Snyder, Jeremy (2015): “'It was the best decision of my life': A thematic content analysis of former medical tourists' patient testimonials". BMC Medical Ethics 16/8. doi: 10.1186/1472-6939-16-8.

Hovland, Carl I./Weiss, Walter (1951): "The influence of source credibility on communication effectiveness". Public Opinion Quarterly 15/4: 635-650. doi: 10.1086/266350.

Huber, Johannes/Ihrig, Andreas/Peters, Tim/Huber, Christian G./Kessler, Anja/Hadaschik, Boris/Pahernik, Sascha/Hohenfellner, Markus (2011): "Decision-making in localized prostate cancer: Lessons learned from an online support group". BJU International 107/10: 1570-1575. doi: 10.1111/j.1464-410X.2010.09859.x.

Huh, Jina/Liu, Leslie S./Neogi, Tina/Inkpen, Kori/Pratt, Wanda (2014): "Health vlogs as social support for chronic illness management”. ACM Transactions on Computer-Human Interaction (TOCHI) 21/4. doi: 10.1145/2630067.

James, Nick/Daniels, Harry/Rahman, Rubina/McConkeyt, Christopher/Derry, Jan/Young, Annie (2007): "A study of information seeking by cancer patients and their carers". Clinical Oncology 19/5: 56-62. doi: 10.1016/j.clon.2007.02.005.

Kouper, Inna (2010): “The pragmatics of peer advice in a LiveJournal community". Language@Internet 7: article 1.

Liu, Leslie S./Huh, Jina/Neogi, Tina/Inkpen, Kopri/Pratt, Wanda (2013): "Health vloggerviewer interaction in chronic illness management". In: Proceedings of the SIGCHI conference on Human factors in computing systems. New York, ACM Press: 49-58. doi: 10.1145/2470654.2470663.

Locher, Miriam A./Hoffmann, Sebastian (2006): "The emergence of the identity of a fictional expert advice-giver in an American internet advice column". Text \& Talk 26/1: 69-106. doi: 10.1515/TEXT.2006.004. 
Mazanderani, Fadhila/O'Neill, Brendan/Powell, John (2013): “'People power' or 'pester power'? YouTube as a forum for the generation of evidence and patient advocacy". Patient Education and Counseling 93/3: 420-425. doi: 10.1016/j.pec.2013.06.006.

McNeill, Andrew/Sillence, Elizabeth (under review): "Sharing experiences of male pattern baldness treatments online: Understanding why users share videos and advice about hair loss on Youtube".

Metzger, Miriam J./Flanagin, Andrew J. (2013). "Credibility and trust of information in online environments: The use of cognitive heuristics". Journal of Pragmatics 59: 210-220. doi: 10.1016/j.pragma.2013.07.012.

Metzger, Miriam J./Flanagin, Andrew J. (2015): "Psychological approaches to credibility assessment online". In: Sundar, S. Shyam (ed.): Handbook of the Psychology of Communication Technology. Hoboken/NJ, Wiley-Blackwell: 445-466.

Mo, Phoenix K. H./Coulson, Neil S. (2012): "Developing a model for online support group use, empowering processes and psychosocial outcomes for individuals living with HIV/AIDS”. Psychology \& Health 27/4: 445-459. doi: 10.1080/08870446.2011.592981.

Phua, Joe (2016): "The effects of similarity, parasocial identification, and source credibility in obesity public service announcements on diet and exercise self-efficacy". Journal of Health Psychology 21/5: 699-708. doi: 10.1177/1359105314536452.

Rapley, Tim (2008): "Distributed decision making: The anatomy of decisions-in-action". Sociology of Health and Illness 30/3: 429-444. doi: 10.1111/j.1467-9566.2007.01064.x.

Rogers, Everett M. (2003): Diffusion of Innovations. New York: Free Press.

Sillence, Elizabeth (2010): "Seeking out very like-minded others: Exploring trust and advice issues in an online health support group". International Journal of Web Based Communities 6: 376-394. doi: 10.1504/IJWBC.2010.035840.

Sillence, Elizabeth (2013): "Giving and receiving peer advice in an online breast cancer support group". Cyberpsychology, Behavior and Social Networking 16/6: 480-485. doi: 10.1089/cyber.2013.1512.

Sillence, Elizabeth/Bussey, Lauren (2017): “Changing hospitals, choosing chemotherapy and deciding you've made the right choice: Understanding the role of online support groups in different health decision-making activities". Patient Education and Counseling 100/5: 994-999. doi: 10.1016/j.pec.2016.12.004.

Sillence, Elizabeth/Hardy, Claire/Briggs, Pam (2013). "Why don't we trust health websites that help us help each other?: An analysis of online peer-to-peer healthcare”. In: Proceedings of the 5th Annual ACM Web Science Conference. New York, ACM: 396-404. doi: 10.1145/2464464.2464488.

Sillence, Elizabeth/Hardy, Claire/Harris, Peter R./Briggs, Pam (2014): "Modelling patient engagement in peer-to-peer healthcare". In: Proceedings of Companion Publication of the 23rd International Conference on World Wide Web. New York, ACM: 481-486. doi: 10.1145/2567948.2576937.

Sillence, Elizabeth/Hardy, Claire/Briggs, Pam/Harris, Peter R. (2015): "How do carers of people with multiple sclerosis engage with websites containing the personal experiences of other carers and patients?". Health Informatics Journal 22/4: 1045-1054. doi: $10.1177 / 1460458215607938$. 
Swann, William B./Read, Stephen, J. (1981): "Self-verification processes: How we sustain our self-conceptions". Journal of Experimental Social Psychology 17: 351-372. doi: 10.1016/0022-1031(81)90043-3.

Tebes, Jacob K. (2005): "Community science, philosophy of science, and the practice of research". American Journal of Community Psychology 35/3-4: 213-230. doi: 10.1007/ s10464-005-3399-x.

Vance, Karl/Howe, William/Dellavalle, Robert P. (2009): "Social internet sites as a source of public health information”. Dermatologic Clinics 27/2: 133-136. doi: 10.1016/j.det.20 08.11.010.

van Uden-Kraan, Cornelia F./Drossaert, Constance H. C./Taal, Erik/Seydel, Erwin/van de Laar, Mart (2008): "Self-reported differences in empowerment between lurkers and posters in online patient support groups". Journal of Medical Internet Research 10/2: e18. doi: 10.2196/jmir.992.

Wartella, Ellen/Rideout, Vicky/Montague, Heather/Beaudoin-Ryan, Leanne/Lauricella, Alexis (2016): "Teens, health and technology: A national survey". Media and Communication 4/3. doi: 10.17645/mac.v4i3.515.

Wotanis, Lindsey/McMillan, Laurie (2014): "Performing gender on YouTube: How Jenna Marbles negotiates a hostile online environment". Feminist Media Studies 14/6: 912-928. doi: 10.1080/14680777.2014.882373.

Ziebland, Sue/Wyke, Sally (2012): "Health and illness in a connected world: How might sharing experiences on the internet affect people's health?". Millbank Quarterly 90: 219-49. doi: $10.1111 / \mathrm{j} .1468-0009.2012 .00662 . x$.

\section{Bionote}

Elizabeth Sillence is a Senior Lecturer in Psychology at Northumbria University, UK and is a founding member of the PaCT (Psychology and Communication Technologies) Lab (www.pactlab.org.uk/). She has a background in psychology and ergonomics and a $\mathrm{PhD}$ in Human Computer Interaction. Liz's research interests are focused on trust and online interactions typically within a health context. This research centres on understanding the factors that impact upon trust and engagement with online health resources. This has often involved either the study of advice exchange within web based forums or has centred on the experiences of people searching for information and advice for themselves or for a friend or family member. She is currently investigating the use of online personal experiences of health and wellbeing in relation to decision making. Liz also studies the development and maintenance of online communities and explores the ethical issues associated with mobile technologies. She has worked on a number of funded projects exploring trust and engagement in health and security settings and has published widely on the issues of trust, e-health and online communities. 